\title{
ASSESSMENT OF ALTERNATIVE DIVERTOR CONFIGURATIONS AS AN EXHAUST SOLUTION FOR DEMO
}

\author{
H. REIMERDES ${ }^{1 *}$, R. AMBROSINO 2 , P. INNOCENTE ${ }^{3}$, A. CASTALDO ${ }^{2}$, P. CHMIELEWSKI $^{4}$, \\ G. DI GIRONIMO ${ }^{2}$, S. MERRIMAN ${ }^{5}$, V. PERICOLI-RIDOLFINI ${ }^{4}$, L. AHO-MANTILLA ${ }^{6}$, R. ALBANESE $^{2}$,

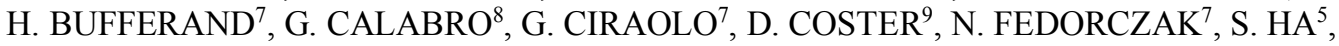 \\ R. KEMBLETON $^{10}$, K. LACKNER ${ }^{9}$, V.P. LOSCHIAVO $^{2 \dagger}$, T. LUNT ${ }^{9}$, D. MARZULLO ${ }^{2 \ddagger}$, R. MAURIZIO $^{1}$, \\ F. MILITELLO ${ }^{5}$, G. RAMOGIDA ${ }^{11}$, F. SUBBA ${ }^{12}$, S. VAROUTIS $^{13}$, R. ZAGÓRSKI ${ }^{14}$ AND H. ZOHM
}

${ }^{1}$ Ecole Polytechnique Fédérale de Lausanne (EPFL), Swiss Plasma Center (SPC), Lausanne, Switzerland

${ }^{2}$ Consorzio CREATE, Universita' degli Studi di Napoli Federico II, Naples, Italy

${ }^{3}$ Consorzio RFX, Padova, Italy

${ }^{4}$ Institute for Plasma Physics and Laser Microfusion (IPPLM), Warsaw, Poland

${ }^{5} \mathrm{CCFE}$, Culham Science Centre, Abingdon, UK

${ }^{6}$ VTT Technical Research Center of Finland Ltd, Espoo, Finland

${ }^{7}$ IRFM, CEA Cadarache, St. Paul-lez-Durance, France

${ }^{8}$ Department of Economics, Engineering, Society and Business Organization, University of Tuscia, Viterbo, Italy

${ }^{9}$ Max-Planck Institute for Plasma Physics, Garching, Germany

${ }^{10}$ EUROfusion PMU, Boltzmannstr. 2, Garching, Germany

${ }^{11}$ ENEA, Frascati, Italy

${ }^{12}$ Dipartimento Energia, Politecnico di Torino, Turin, Italy

${ }^{13}$ KIT, Karlsruhe, Germany

${ }^{14}$ National Centre for Nuclear Research, Otwock, Poland

\begin{abstract}
Plasma exhaust has been identified as a major challenge towards the realisation of magnetic confinement fusion. To mitigate the risk that the single null divertor (SND) with a high radiation fraction in the scrape-of-layer (SOL) adopted for ITER will not extrapolate to a DEMO reactor, the EUROfusion consortium is assessing potential benefits and engineering challenges of alternative divertor configurations. Alternative configurations that could be readily adopted in a DEMO design include the X divertor (XD), the Super-X divertor (SXD), the Snowflake divertor (SFD) and the double null divertor (DND). The flux flaring towards the divertor target of the XD is limited by the minimum grazing angle at the target set by gaps and misalignments. The characteristic increase of the target radius in the SXD is a trade-off with the increased TF coil volume, but, ultimately, also limited by forces onto coils. Engineering constraints also limit XD and SXD characteristics to the outer divertor leg with a solution for the inner leg requiring up-down symmetric configurations. Capital cost increases with respect to a SND configuration are largest for SXD and SFD, which require both significantly more poloidal field coil conductors and in the case of the SXD also more toroidal field coil conductors. Boundary models with increasing degrees of complexity have been used to predict the beneficial effect of the alternative configurations on exhaust performance. While all alternative configurations should decrease the power that must be radiated in the outer divertor, only the DND and possibly the SFD also ease the radiation requirements in the inner divertor. These decreases of the radiation requirements are however expected to be small making the ability of alternative divertors to increase divertor radiation without excessive core performance degradation their main advantage. Initial 2D fluid modeling of argon seeding in XD and SFD configurations indicate such advantages over the SND, while results for SXD and DND are still pending. Additional improvements, expected from increased turbulence in the low poloidal field region of the SFD also remain to be verified. A more precise comparison with the SND as well as absolute quantitative predictions for all configurations requires more complete physics models that are currently only being developed.
\end{abstract}

\section{INTRODUCTION}

The European roadmap for fusion energy [1,2] has identified a reliable solution for heat and particle exhaust as one of the main challenges towards the realisation of magnetic confinement fusion. Since power exhaust scales unfavourably with the size of the device, a reactor based on the tokamak concept must likely harness an even greater heat flux than ITER. Simultaneously, the higher particle and neutron fluences impose more stringent constraints on the plasma facing components and the admissible erosion. In the current European baseline scenario

\footnotetext{
*Email: holger.reimerdes@epfl.ch

†Present address: Università degli Studi del Sannio, Dipartimento di Ingegneria, Benevento, Italy

Fresent address: University of Trieste, Department of Engineering and Architecture, Trieste, Italy
} 
for a DEMO reactor, which foresees a single null magnetic configuration, this must be achieved by an even greater radiative power exhaust [3]. However, it is, as discussed in Section 2, uncertain whether greater divertor radiation is compatible with the energy confinement required in a reactor $[4,5]$ and whether transients can be sufficiently suppressed to avoid any damage of the divertor targets. To mitigate the risk that the baseline scenario adopted for ITER will not extrapolate to DEMO, the EUROfusion consortium is assessing the potential benefits and the engineering challenges of alternative divertor configurations as an exhaust solution for DEMO.

Several decades of divertor research have resulted in many configurations and concepts that may be considered as alternatives to the conventional divertor [6]. Among these configurations, a reduced set of basic geometry variations and corresponding divertor concepts that rely on the same physics basis as the baseline solution and can readily be adopted in a DEMO design is identified, and the underlying physics mechanisms described in Section 3. Alternative concepts generally increase the complexity of the device and their realization may exceed available technological capabilities. The extent of the geometric variations that could be attainable using only modest extrapolations of currently available technologies is evaluated in Section 4. While the range of achieved geometric variations may not be optimal, they are indicative of the achievable variations within the given constraints. Divertor models with increasing degrees of sophistication are then used in Section 5 to project the geometric variations into divertor performance improvements. To decrease the effect of systematic errors, these are compared to predictions for the baseline solution. The conclusions of the assessment are presented in Section 6 .

\section{THE PLASMA EXHAUST CHALLENGE}

The EU roadmap foresees a demonstration fusion power plant (DEMO) that will follow ITER with the capability of generating several hundred MW of net electricity [7]. Aiming at a net electric power output of $500 \mathrm{MW}$ with a conventional aspect ratio $(A=3.1)$ tokamak the system code PROCESS is used to identify the main reactor parameters. Assuming a maximum magnetic field at the location of the toroidal field coils of $11.9 \mathrm{~T}$ limited by excessive forces onto the coils, an $\mathrm{H}$-mode confinement factor $H_{98}=1.1$, a density that exceeds the Greenwald density by $20 \%$ and a normalized beta $\beta_{\mathrm{N}}=2.6$ results in a device with a major radius of $8.8 \mathrm{~m}$, an on-axis magnetic field of $5.8 \mathrm{~T}$ and a plasma current of $20.3 \mathrm{MA}$, Table 1. Correcting for core brems- and synchrotron radiation losses the plasma is heated with $P_{\text {heat }}=300 \mathrm{MW}$.

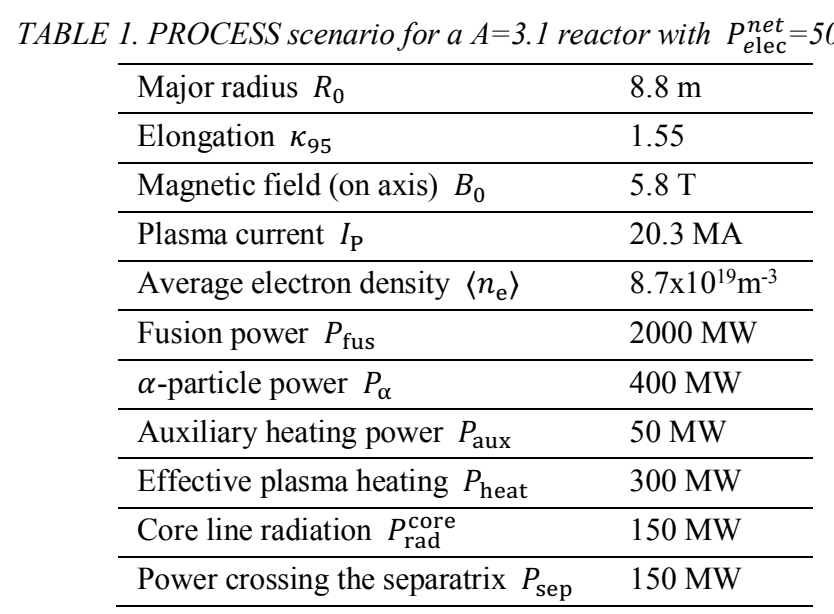

The empiric scaling of the L-H threshold [8] requires that at least $135 \mathrm{MW}$ of the heating power cross the LCFS in charged particles to access H-mode confinement. The L-H threshold together with a $10 \%$ margin sets the minimum $P_{\text {sep }}=150 \mathrm{MW}$ that must be exhausted in the divertor, with the remaining $150 \mathrm{MW}$ exhausted by line radiation in the closed field line region, Table 1. In addition to removing power, the divertor must also exhaust $7 \times 10^{20} \mathrm{He}$ atoms/s that are generated in the fusion reactions. In the baseline scenario [3] the plasma exhaust relies on an extrapolation of the ITER solution [9] characterised by high divertor radiation obtained through impurity seeding and operation in a highly detached divertor regime, while maintaining a high neutral pressure in the pump ducts.

An empiric scaling [10] and a heuristic drift-based model [11] of the scrape off layer (SOL) width $\lambda_{\mathrm{q}}$ in attached scenarios predict values of approximately $1.0 \mathrm{~mm}$ for the outboard midplane of the considered reactor scenario, Table 1. Assuming a typical 1:2 power distribution between the inner and outer divertor the expected upstream parallel heat flux towards the outer divertor is $q_{\|, \mathrm{u}} \approx 5 \mathrm{GW} / \mathrm{m}^{2}$. The unmitigated parallel heat flux at the target, $q_{\|, t}^{*}$, would be even somewhat higher as the cross section of flux tubes near the targets is smaller due to the larger magnetic field at the outer target of the conventional single null configuration, Table 2. Such a high heat flux must 
be reconciled with technological solutions for the target, which impose limits most notably on the maximum heat flux onto the target surface and on the maximum electron temperature at the target.

The heat flux onto the target is limited to prevent excessive temperatures of any target component, which may cause melting or embrittlement but also radiation creep. Materials must, in particular, be chosen to be compatible with the high neutron fluence in the DEMO divertor. A typical value for the maximum heat flux onto the surface of a target made of reactor relevant materials is $10 \mathrm{MW} / \mathrm{m}^{2}$ [12]. Fortunately, the grazing angle between magnetic field lines and the target surface, $\alpha_{\mathrm{t}}$, is small, which reduces the component of the parallel plasma heat flux that is perpendicular to the target surface,

$$
q_{\perp, \mathrm{t}}=q_{\|, \mathrm{t}} \sin \alpha_{\mathrm{t}}
$$

The value of $\alpha_{\mathrm{t}}$ can be reduced by decreasing the poloidal field at the target and, hence, increasing the poloidal flux expansion $f_{\mathrm{x}, \mathrm{t}} \mathrm{t}^{\mathrm{a}}$ and by tilting the target in the poloidal direction with respect to the separatrix. However, the need to assemble the divertor targets out of small building blocks leads to poloidal gaps and leading edges that must be shielded. Such shielding is typically achieved through chamfering of the target surface, which, together with manufacturing tolerances, impose a minimum value for $\alpha_{\mathrm{t}}$. A similar constraint arises also from the need to control the position of the strike line. The ITER design resulted for example in $\alpha_{\mathrm{t}} \sim 3^{\circ}\left(+1^{\circ}\right.$ bevel of the monoblocks) [13]. The chamfering of the target surface also reduces the maximum heat flux in charged particles perpendicular to a toroidally symmetric target surface, $q_{\perp, \mathrm{t}}^{\max }$, with respect to the maximum heat flux capability of the target. A further reduction arises from the need for a margin to handle target misalignments as well as remove heat deposited by radiation and neutral particles. Assuming a maximum axisymmetric plasma heat removal at the target of $q_{\perp, \mathrm{t}}^{\max }=5 \mathrm{MW} / \mathrm{m}^{2}$ and a minimum angle of $\alpha_{\mathrm{t}}=3^{\circ}$ the maximum parallel heat flux at the target is $q_{\|, \mathrm{t}}^{\max }=100 \mathrm{MW} / \mathrm{m}^{2}$. Even assuming that divertor broadening reduces the unmitigated peak heat flux by a factor of three (to $2.2 \mathrm{GW} / \mathrm{m}^{2}$ ), at least $2.1 \mathrm{GW} / \mathrm{m}^{2}$ corresponding to $96 \%$ of the power crossing the LCFS in charged particles must be dissipated along field lines in the divertor, Table 2. Such a large dissipation must be achieved through deliberate seeding of impurities in the divertor, with argon (Ar) having been identified as a possible species [7]. Achieving such a level of radiative power exhaust along magnetic field lines in the divertor, $\Delta q_{\text {rad,div }}$, will, however, require an increase of the seed impurity concentration in the divertor over today's devices as well as ITER [4,5], which may not be compatible with core performance. Leakage of seed impurities from the divertor into the plasma core must be sufficiently small to avoid excessive core radiation as well as excessive fusion fuel dilution. The exact limits depend on the scenario. In the reference scenario the core radiation arising from divertor impurity seeding must be less than $150 \mathrm{MW}$ and compatible with $H_{98}=1.1$ and the additional dilution of the fusion fuel must not reduce the alpha heating below $400 \mathrm{MW}$.

An additional limit is introduced by sputtering of the target material [14], which increases with the electron temperature at the target, $T_{\mathrm{e}, \mathrm{t}}$. The sputtering has to be sufficiently low to avoid a reduction of the lifetime of the divertor and to avoid an intolerable influx of heavy impurities that would degrade core performance. With tungsten (W) being the most promising material for the divertor target armor, the maximum value of $T_{\mathrm{e}, \mathrm{t}}$ is set by sputtering of $\mathrm{W}$ through impact of seed impurity ions to values below $5 \mathrm{eV}$.

The value of $T_{\mathrm{e}, \mathrm{t}}$ is closely linked with the heat transfer through the plasma sheath at the target,

$$
q_{\|, \mathrm{t}} \approx\left(\gamma_{\text {sheath }} \sqrt{k_{\mathrm{B}} T_{\mathrm{e}, \mathrm{t}}}+\frac{E_{\mathrm{pot}}}{\sqrt{k_{\mathrm{B}} T_{\mathrm{e}, \mathrm{t}}}}\right) \frac{p_{\mathrm{t}}}{\sqrt{2 m_{\mathrm{i}}}},
$$

where $\gamma_{\text {sheath }}$ is the sheath heat transmission factor, $E_{\text {pot }}$ the potential energy and $p_{\mathrm{t}}$ the plasma pressure at the target. For fixed plasma pressure at the target, $q_{\|, \mathrm{t}}$ has a minimum for $T_{\mathrm{e}, \mathrm{t}} \sim 2-3 \mathrm{eV}$. As the upstream pressure is poised to increase with the higher heat flux and longer connection length in a reactor compared to today's devices and ITER, obtaining the same acceptable values for $q_{\|, \mathrm{t}}$ and $T_{\mathrm{e}, \mathrm{t}}$ will require a larger pressure loss along SOL field lines. Since the pressure loss increases with lower $T_{e, t}$ [15] DEMO must operate at lower $T_{e, t}$ than today's devices. The lower value of $T_{\mathrm{e}, \mathrm{t}}$ raises the concern that the operating regime is not stable as well as that the neutral pressure in the divertor and, hence, in the pump ducts may drop.

The prediction of the magnitude of the exhaust challenge in DEMO is subject to large uncertainties. For example, recent gyro-kinetic transport simulations suggest that the empiric scaling of $\lambda_{\mathrm{q}}$ does not extend to ITER and DEMO, where turbulence is predicted to surpass magnetic drifts as the main cross-field transport channel, increasing $\lambda_{\mathrm{q}}$ to values of the order $5 \mathrm{~mm}$ [16]. While such a five-fold increase of $\lambda_{\mathrm{q}}$ would still require a divertor radiation fraction, $f_{\text {rad,div }}^{\text {req }}$, as high as $\sim 80 \%$ (scenario $\# 2$ in Table 2 ), the available radiation volume increases, which is reflected in a corresponding reduction of the heat flux that must be mitigated along field lines, $\Delta q_{\|, \text {rad,div }}^{\text {req }}$ The exhaust challenge may also be eased by prospective advances of engineering capabilities and reduced manufacturing tolerances. A hypothetical decrease of the minimum grazing angle at the target to $\alpha_{\mathrm{t}} \sim 1.5^{\circ}$

§The poloidal flux expansion is here defined as the ratio of the flux surface separation at the target and upstream. 
would double its capability to remove parallel heat flux, $q_{\|, \mathrm{t}}^{\max }$, albeit only with modest consequences for the required radiative heat exhaust in the divertor (scenario \#3 in Table 2). While decreasing $\alpha_{\mathrm{t}}$ would help establishing a tolerable target heat flux, the resulting larger parallel heat flux magnifies the challenge to decrease $T_{\mathrm{e}, \mathrm{t}}$ to tolerable values. The assumed margin between the heat removal capability of the target and the maximum plasma heat removal of $5 \mathrm{MW} / \mathrm{m}^{2}$ may not be sufficient for the high level of divertor radiation. Reducing the limit for plasma heat exhaust to $2.5 \mathrm{MW} / \mathrm{m}^{2}$, has again only modest consequences for the absolute value of radiative heat exhaust (scenario \#4 in Table 2). While all considered scenarios must rely on an unprecedented level of radiative heat exhaust, possible variations of the SOL width have clearly the largest leverage over the magnitude of the challenge.

TABLE 2. Parameters describing the exhaust challenge and their dependence on the assumed SOL width, $\lambda_{q}$, field line angle at the target, $\alpha_{t}$, and plasma heat removal capability of the target, $q_{\perp, t}^{\max }$. Numbers in bold highlight changes with respect to the reference scenario.

\begin{tabular}{|c|c|c|c|c|c|c|c|c|}
\hline & Scenario & $q_{\|, \mathrm{u}}$ & $q_{\|, \mathrm{t}}^{*}$ & $q_{\|, \mathrm{t}}^{* *}$ & $q_{\perp, \mathrm{t}}^{\max }$ & $q_{\|, \mathrm{t}}^{\max }$ & $\Delta q_{\|, \mathrm{rad}, \mathrm{div}}^{\mathrm{req}}$ & $f_{\text {rad,div }}^{\text {req }}$ \\
\hline \# & assumptions & {$\left[\mathrm{MW} / \mathrm{m}^{2}\right]$} & {$\left[\mathrm{MW} / \mathrm{m}^{2}\right]$} & {$\left[\mathrm{MW} / \mathrm{m}^{2}\right]$} & {$\left[\mathrm{MW} / \mathrm{m}^{2}\right]$} & {$\left[\mathrm{MW} / \mathrm{m}^{2}\right]$} & {$\left[\mathrm{MW} / \mathrm{m}^{2}\right]$} & \\
\hline 1 & Described above & 4700 & 6600 & 2200 & 5.0 & 96 & 2100 & 0.96 \\
\hline 2 & Increase $\lambda_{\mathrm{q}} \sim 5 \mathrm{~mm}$ & 910 & 1300 & 430 & 5.0 & 96 & 330 & 0.78 \\
\hline 3 & Decrease $\alpha_{\mathrm{t}} \sim 1.5^{\circ}$ & 4700 & 6600 & 2200 & 5.0 & 190 & 2000 & 0.91 \\
\hline 4 & $\begin{array}{l}\text { Decrease } \\
q_{\perp, \mathrm{t}}^{\max }=2.5 \mathrm{MW} / \mathrm{m}^{2}\end{array}$ & 4700 & 6600 & 2200 & 2.5 & 48 & 2150 & 0.98 \\
\hline
\end{tabular}

Plasma exhaust in ITER represents a significant step towards DEMO and experiments in ITER will ultimately test whether the conventional single null divertor (SND) with a high radiation fraction in the SOL will extrapolate to a reactor. To mitigate the risk that the baseline will not extrapolate to DEMO, the potential benefits and the engineering challenges of alternative divertor configurations are assessed.

\section{ALTERNATIVE DIVERTOR CONFIGURATIONS}

To avoid significant delays in the European effort to design a DEMO reactor [17,18], the assessment only considers alternative configurations that rely on the same core physics, including $\mathrm{H}$-mode confinement and detached divertor operation, as the baseline scenario. The considered configurations include a X, Super-X, Snowflake and Double-Null divertor, all of which have already been realised experimentally. Key aspects of these configurations also apply to other concepts, such as long-legged, tightly baffled, divertors, the X-point target divertor and the tripod divertor.

\section{1. $\mathrm{X}$ divertor}

The X divertor (XD) concept [19] relies on a flaring of the poloidal flux towards the target with two main consequences for the plasma exhaust. Firstly, a larger flux expansion at the target, $f_{\mathrm{x}, \mathrm{t}}$, increases the wetted area, albeit by decreasing the grazing angle of field lines at the target, $\alpha_{\mathrm{t}}$. While the same increase can be obtained by a poloidal tilt of the target, it is suggested that a higher flux expansion facilitates the control of the strike point location possibly providing a lower grazing angle at the target [20]. Secondly, flaring reduces the interaction area of neutrals towards the X-point thereby introducing a mechanism that keeps the neutral interaction region close to the target [20]. This may increase the operational range where the detachment front, and hence the region of high neutral pressure, remains close to the target decreasing demands on the detachment control system. In addition, increasing $f_{\mathrm{x}, \mathrm{t}}$ increases the connection length, $L_{\|}$, which should lower the detachment threshold. A beneficial effect on the detachment threshold beyond the increase of $L_{\|}$is supported by fluid modelling using the SOLPS code [21].

The XD configuration was realised in ASDEX [22] well before the formulation of the XD divertor concept. The plasma exhaust behaviour of the XD configuration was subsequently compared to the SND in TCV [23, 24] and DIII-D [21], but the comparison only showed a negligible or small beneficial effect.

\subsection{Super-X divertor}

The Super-X divertor (SXD) concept [25] extends the XD concept to toroidal flux flaring by increasing the major radius of the target, $R_{\mathrm{t}}$. The increase of $R_{\mathrm{t}}$ increases the cross-sectional area of flux tubes and, thereby, decrease $q_{\|, \mathrm{t}}$ even in the absence of cross-field losses. At constant grazing angle of field lines at the target, $\alpha_{\mathrm{t}}$, the $q_{\|, \mathrm{t}} \propto$ $R_{\mathrm{t}}^{-1}$ dependence is exact, which is equivalent with a linear increase of the wetted area. This decreases the peak 
heat flux that must be mitigated and lowers the detachment threshold. The increase of the cross-sectional area of flux tubes towards the target also results in an inverse gradient in the parallel heat flux, which should stabilise the radiation-condensation instability. A movement of the cool radiation front along the divertor leg towards the Xpoint would be encountered with an increase in $q_{\|}$, which opposes further radiation cooling and, hence, the movement of the front [26]. The difference in $q_{\|}$between target and X-point, thereby, increases the detachment window [27]. The increase of the target radius in the SXD can be combined with an increase of the poloidal flux expansion, as planned in MAST-upgrade [28], or even with an additional null point along the divertor leg as proposed in the X-point target divertor concept [29]. The decrease of the poloidal field would significantly increase the connection length and, thereby further reduce the detachment threshold. An increase in $R_{\mathrm{t}}$ usually comes with a longer divertor leg, $L_{\mathrm{p}}$, which should adjust the balance between parallel and cross-field transport and result in a broader width of the power carrying channel, $\lambda_{\mathrm{q}}$. An increase of $R_{\mathrm{t}}$ should also facilitate the shielding of high heat flux components from neutron irradiation, increasing the choice and capabilities in the materials that can be used. A beneficial effect has also been seen in fluid simulations using the UEDGE [30] as well as the SOLPS code [31].

Significant variations of the outer target radius were experimentally obtained in DIII-D [32] and TCV [24], but in both experiments variations of the target geometry appear to mask the effect of $R_{\mathrm{t}}$.

\subsection{Snowflake divertor (SFD)}

The snowflake divertor concept $[33,34]$ is based on a second order null point, where divertor coils simultaneously cancel the poloidal field, $B_{\mathrm{p}}$, and its gradients, $\nabla B_{\mathrm{p}}$, and which leads to a characteristic hexagonal symmetry of the separatrix. In the vicinity of a second order null, $B_{\mathrm{p}}$ is lower than in a conventional X-point, which increases connection length and SOL volume. This increase is largest closest to the separatrix, where the unmitigated heat flux is highest. An increased $L_{\|}$is expected to facilitate access to detachment. It is furthermore hypothesised that the decrease of the poloidal field increases turbulent cross-field transport or even macroscopic magnetohydrodynamic instabilities $[35,36]$ that broaden $\lambda_{\mathrm{q}}$, thereby further facilitating access to detachment. In a SFD, the poloidal flux is re-concentrated towards the target. Following the reasoning for the XD, Section 3.1, this may enhance the movement of a detachment front towards the null-region. It is, therefore, likely that the operating regime of a SFD reactor will resemble X-point radiators [37]. In such a regime, the SFD may have a smaller impact on core confinement than a SND with the low $B_{\mathrm{p}}$ region extending further into the region of closed field lines, where it may support higher poloidal gradients.

Due to inevitable deviations from the exact current distribution in the plasma, the poloidal field coils and passive structures, a real snowflake configuration features two nearby X-points with only one "primary" X-point with a non-zero $\nabla B_{\mathrm{p}}$ determining the separatrix. Depending on the location of the secondary X-point in the private or common flux region one distinguishes snowflake-plus (SFD+) and snowflake-minus (SFD-) configurations. An increased distance generally increases $\nabla B_{\mathrm{p}}$ at the primary X-point, which weakens the main advantages of the SFD, and leads to a set of proximity conditions $[34,38]$. The placement of the secondary null in the SOL of the outer divertor, referred to as a low-field side (LFS) SFD-, may however even be desirable as it can decrease the peak parallel heat fluxes where it is most needed [39, 40]. SFD- configurations are, therefore, usually parameterised by the outboard-midplane ('upstream') distance of the flux surface that contains the secondary Xpoint from the separatrix, $d R_{\mathrm{u}, \mathrm{X} 2}$.

The SFD configuration was realised in TCV [41], NSTX [42] and DIII-D [43], but experimental observations are difficult to extrapolate to a reactor as the geometric modifications of the SOL depend strongly on the ratio of $\lambda_{\mathrm{q}}$ and the device dimensions, e.g. $R_{0}[44]$.

\subsection{Double null divertor}

The double null divertor (DND) is an up-down symmetric configuration with first order X-points at the top and bottom and corresponding divertors. As the transport across the LCFS has a strong ballooning character, heat and particles are predominantly exhausted to the outer targets, which have a larger $R_{\mathrm{t}}$ than the inner targets and, hence, as discussed in Section 3.2 for the SXD concept, lower peak heat fluxes at the targets. The DND is, therefore, foremost a solution for the inner divertor of the baseline configuration. An additional advantage is an extremely quiescent and narrow inner SOL [45], with strongly reduced heat flux onto the inner wall that reduces the required breeding blanket armour. It may also facilitate HFS RF coupling [29].

Double-null configurations have been realized in many diverted tokamaks starting with T-12 [46]. The ability to control the power distribution between the upper and lower targets by magnetic balance is well documented (e.g. in MAST [47]).

It may also be necessary to extend the DND concept to alternative configurations, if they cannot protect the inner divertor target. This may be the case for realisable XD and SXD implementations in DEMO, discussed in Section 4.2 , or for configurations that intrinsically favour the outer divertor such as possibly LFS SFD- variants. 


\section{REALISATION IN A DEMO SIZE DEVICE}

Any of the assessed alternative configurations, discussed in Section 3, will increase the complexity of the magnetic configuration and, thereby, the engineering challenge and cost of a power plant. This assessment includes a study of whether the divertor concepts may be realised in a DEMO that uses presently available technologies and identifies limits to attainable geometric variations [48]. A key technology is conventional superconductors, which require dedicated winding facilities and exclude interlinked coils. The assessment, therefore, requires that the poloidal field (PF) coils must be located outside the toroidal field (TF) coils. A maximum magnetic field at the conductor is set to $12.5 \mathrm{~T}$ and the current density in the coils limited to $12.5 \mathrm{MA} / \mathrm{m}^{2}$. Vertical forces onto a single $\mathrm{PF}$ coil must not exceed $450 \mathrm{MN}$ and onto the entire central solenoid (CS) $300 \mathrm{MN}$. The maximum separation force in the CS must not exceed $350 \mathrm{MN}$. All configurations are conceived with $18 \mathrm{TF}$ coils that are sized to limit the TF ripple to $0.6 \%$. Each divertor target is tilted in the poloidal plane with the target surface and the separatrix forming an angle $\beta_{\mathrm{t}}$. The value of $\beta_{\mathrm{t}}$ is chosen for separatrix field lines to intersect the (toroidally symmetric) targets with the same grazing angle, $\alpha_{\mathrm{t}}$, in all configurations and the tilt direction to 'close' the divertor. The reference value for $\alpha_{\mathrm{t}}$ is set to $1.5^{\circ}$, which is a factor of two below the ITER value. Such a choice relies on significant advances over presently available technologies, but proved to be necessary to realise an X-divertor configuration, introduced in Section 3.1, and include it in the quantitative assessment. While the assumed constraints may not be absolutely correct they characterise the orders of magnitude of the feasible.

The resulting reference and alternative configurations are described in Section 4.1 and 4.2, respectively and are referred to as "2017" configurations. Geometric characteristics that are compared include the connections lengths, $L_{\|}$, and its poloidal projection, $L_{\mathrm{p}}$, evaluated from the outboard midplane to both divertor targets. A lowest order estimation of the cost increase of the alternatives with respect to the reference configuration, based on the increased mass of the superconductors is given in Section 4.3. A revision based in the evolving EU baseline, which most notably included a reduction of the number of TF coils from 18 to 16 , has resulted in "2018" configurations discussed in Section 4.4. The "2018" configurations are also basis for a first investigation of possible repercussions for remote handling of the divertor and blanket modules in Section 4.5, which may affect availability and, thereby, the price of electricity. A rigorous ordering of the cost increases is not possible and further possibly important constraints and cost drivers are listed in Section 4.6.

\subsection{Conventional single null reference configuration}

A SND configuration that meets the scenario parameters used in the system code, Table 1, is obtained using $6 \mathrm{PF}$ coils and a central solenoid consisting of 5 individually powered segments, Fig. 1. The TF coils enclose a volume of $7175 \mathrm{~m}^{3}$, that is 3.5 times the plasma volume, and the forces onto the PF coils remain well below the limits. The parallel connection lengths from the outboard midplane to the inner and outer targets, evaluated on the flux surface with an upstream separatrix distance $d R_{\mathrm{u}, \mathrm{sep}}=1 \mathrm{~mm}$, are $215 \mathrm{~m}$ and $125 \mathrm{~m}$, respectively. Moderate flux expansions of 5.7 and 3.8 at the inner and outer targets allow for a 'closed' divertor target configuration with the poloidal angle between separatrix and the target, $\beta_{\mathrm{t}}$, of only $28^{\circ}$ and $20^{\circ}$, respectively.

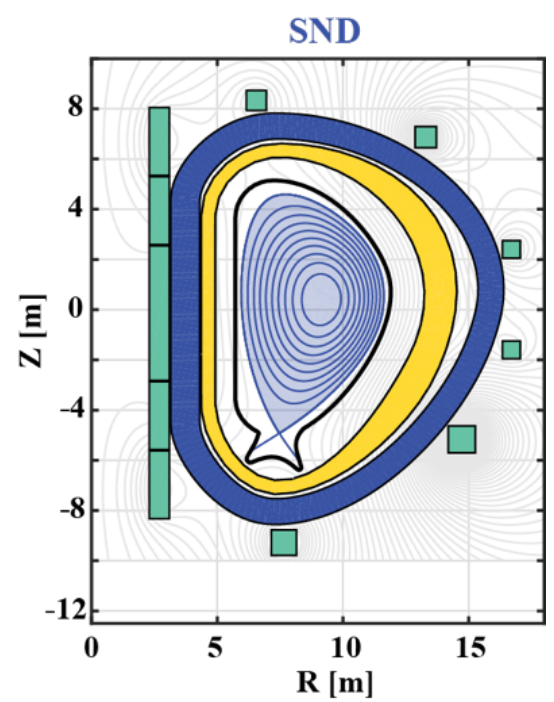

Figure 1. Reference configuration with a Single-Null divertor (SND) (adapted from Fig. 1 in [48]). The 2D description consists of an equilibrium (light blue), a first wall including divertor target (black), a vacuum vessel (yellow), toroidal field coils (dark blue) and poloidal field coils (green). 


\subsection{Alternative configurations}

Alternative configurations with the same plasma and low order shape parameters are realised by iterating between the CREATE-NL code to place PF coils and calculate equilibria and the NOVA code to place first wall, vacuum vessel and TF coils [48]. The first wall maintains a minimum distance of $22.5 \mathrm{~cm}$ to the separatrix. The vacuum vessel allows space for breeding blankets and includes space for additional neutron shielding. The coil placement is optimised to meet the constraints described above and maximise the flat top flux swing. While the range of achieved geometric variations may not be optimal, they should be indicative of the achievable variations under the given constraints.

\subsubsection{X divertor}

The XD configuration can only be realised with poloidal flux flaring at the outer leg, Fig. 2(a) and Table 3, as space constraints prohibit coils that may flare the flux at the inner target. The flux swing for the $\mathrm{XD}$ is significantly reduced and achieving $75 \%$ of the flux swing of the SND requires a highly segmented CS. The flux flaring towards the outer divertor target is limited by the smallest value of $\alpha_{\mathrm{t}}$. To maximise the flux expansion at the target, $f_{\mathrm{x}, \mathrm{t}}$, the target is placed with an angle $\beta_{\mathrm{t}} \sim 90^{\circ}$ with respect to the separatrix. The obtained ratio of the flux expansion at the target and its minimum value along the divertor leg of $f_{\mathrm{x}, \mathrm{t}} / f_{\mathrm{x}}^{\min }=1.3$ quantifies the flaring and is the result of a trade-off with a longer divertor leg and a larger TF coil volume that in the assessed configuration is only marginally larger than for the SND, Table 3. Increasing the leg length and/or relaxing the constraint on $\alpha_{\mathrm{t}}$ even further could lead to a stronger flaring. This would, however, require a detailed study of expected manufacturing tolerances and configuration control capabilities. Flaring could also be increased, if additional poloidal field coils inside the TF coils could be considered. The increase of the outer leg length and the larger flux expansion have a significant effect on $L_{\|}$to the outer target, almost doubling with respect to the SND.

\subsubsection{Super-X divertor}

The SXD configuration can only be realised with an increase in the target radius of the outer target, Fig. 2(b) and Table 3. The obtained target radius corresponds to 1.5 times the $\mathrm{X}$-point radius. The increase in $R_{\mathrm{t}}$ is a trade-off with the TF coil volume that increases by more than $25 \%$ with respect to the SND, but ultimately by forces onto PF coils. The use of "external coils"-only prohibits additional poloidal flux flaring along the outer divertor leg. In the realised configuration, the connection length to the outer target increases by $\sim 75 \%$ with respect to the SND and is almost as large as for the $\mathrm{XD}$. The poloidal tilt of the outer target is strong with $\beta_{\mathrm{t}}=12^{\circ}$, since $f_{\mathrm{x}, \mathrm{t}}$ is small, leading to an extremely 'closed' outer target configuration, Table 3.

\subsubsection{Snowflake divertor}

The SFD configuration can been achieved, within all constraints, with a marginal increase of the TF coil volume with respect to the SND, Fig. 2(c) and Table 3. Similarly to the XD, the flux swing is significantly reduced and achieving $75 \%$ of the flux swing of the SND requires an equally segmented CS. The assessed equilibrium has a $\mathrm{SFD}(+)$ topology with the two X-points being separated by $20 \mathrm{~cm}$, leading to a 25 fold decrease in $\left|\nabla B_{\mathrm{p}, \mathrm{xpt}}\right|$ with respect to the SND. The connection length to the inner and outer targets evaluated on the flux surface with $d R_{\mathrm{u}, \mathrm{sep}}=1 \mathrm{~mm}$ increases by factors of 2.2 and 2.8 , respectively. In addition to the $\mathrm{SFD}(+)$ several $\operatorname{SFD}(-)$ configurations with a range of X-point separations, $d R_{\mathrm{u}, \mathrm{X} 2}$, are generated. The exact location of the secondary Xpoint with respect to the primary Z-point has negligible effects on the coil currents. A SFD(-) configuration with $d R_{\mathrm{u}, \mathrm{X} 2}=1 \mathrm{~mm}$, i.e. smaller than the expected $\lambda_{\mathrm{q}}$, is chosen for the assessment of the divertor performance in Section 5 .

\subsubsection{Double-null divertor}

A DND configuration is realised with a marginal increase of the TF coil volume and a small reduction of the flux swing, Fig. 2(d) and Table 3. The connection length to the outer targets is similar to the outer target of the SND. The poloidal flux expansion at the target is small allowing for a strong poloidal tilt of the outer targets with $\beta_{\mathrm{t}}=13^{\circ}$, Table 2, which leads to extremely 'closed' divertors. The inner targets are not directly connected to the outboard midplane and a corresponding $L_{\|}$, therefore, not defined. 

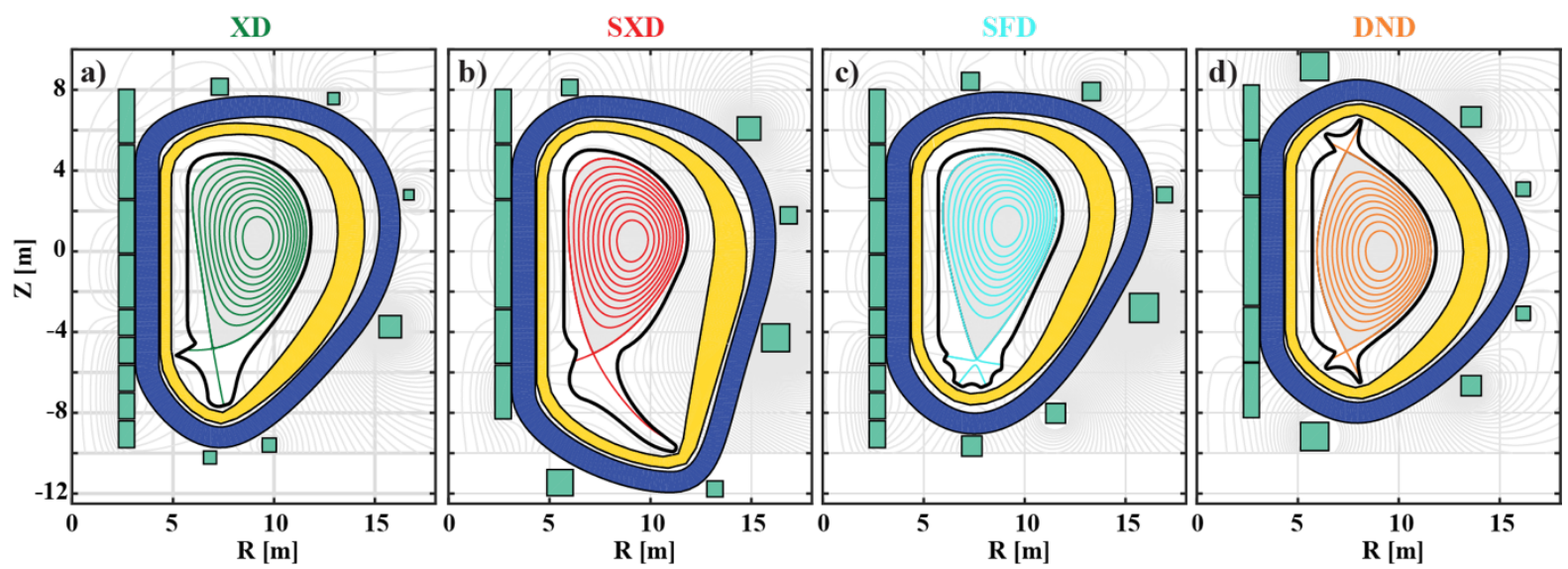

Figure 2. "2017” DEMO configurations featuring (a) a XD, (b) SXD, (c) SFD and (d) DND (adapted from Fig. 1 in [48]).

TABLE 3. Parameters used to estimate the costs and evaluate geometric variations of the "2017" reference SND, Section 3.1, and the corresponding assessed alternative configurations, Section 3.2. An extended version of the table can be found in [48].

\begin{tabular}{|c|c|c|c|c|c|c|c|c|c|c|c|}
\hline & & \multicolumn{2}{|c|}{ SND } & \multicolumn{2}{|c|}{ XD } & \multicolumn{2}{|c|}{ SXD } & \multicolumn{2}{|c|}{$\operatorname{SFD}(+)$} & \multicolumn{2}{|c|}{ DND } \\
\hline \multirow{4}{*}{$\dot{\tilde{\theta}}$} & $V_{\mathrm{TF}} / V_{\text {plasma }}$ & \multicolumn{2}{|c|}{3.50} & \multicolumn{2}{|c|}{3.61} & \multicolumn{2}{|c|}{4.42} & \multicolumn{2}{|c|}{3.57} & \multicolumn{2}{|c|}{3.60} \\
\hline & $L_{\mathrm{TF}}[\mathrm{m}]$ & \multicolumn{2}{|c|}{43.9} & \multicolumn{2}{|c|}{45.9} & \multicolumn{2}{|c|}{50.5} & \multicolumn{2}{|c|}{45.1} & \multicolumn{2}{|c|}{44.4} \\
\hline & $\sum R_{\mathrm{PF}} I_{\mathrm{PF}}^{\max } \quad$ [m·MA $\cdot$ turns] & \multicolumn{2}{|c|}{690} & \multicolumn{2}{|c|}{665} & \multicolumn{2}{|c|}{1016} & \multicolumn{2}{|c|}{970} & \multicolumn{2}{|c|}{744} \\
\hline & Flux swing [Vs] & \multicolumn{2}{|c|}{240} & \multicolumn{2}{|c|}{185} & \multicolumn{2}{|c|}{200} & \multicolumn{2}{|c|}{180} & \multicolumn{2}{|c|}{220} \\
\hline \multirow{6}{*}{ 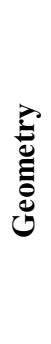 } & $\left|\nabla B_{\mathrm{p}, \mathrm{xpt}}\right|[\mathrm{T} / \mathrm{m}]$ & \multicolumn{2}{|c|}{0.43} & \multicolumn{2}{|c|}{0.32} & \multicolumn{2}{|c|}{0.29} & \multicolumn{2}{|c|}{0.016} & \multicolumn{2}{|c|}{0.56} \\
\hline & & inner & outer & inner & outer & inner & outer & inner & outer & inner & outer \\
\hline & $R_{\mathrm{t}}[\mathrm{m}]$ & 6.54 & 8.29 & 5.64 & 7.54 & 6.22 & 10.8 & 6.10 & 8.86 & 6.61 & 8.14 \\
\hline & $L_{\mathrm{p}}[\mathrm{m}]$ & 18.1 & 8.5 & 17.7 & 10.8 & 17.7 & 13.1 & 18.1 & 9.5 & - & 8.3 \\
\hline & $L_{\|}\left(d R_{\mathrm{u}}=1 \mathrm{~mm}\right)[\mathrm{m}]$ & 215 & 125 & 237 & 236 & 238 & 217 & 464 & 344 & - & 104 \\
\hline & $\beta_{\mathrm{t}}[$ Deg. $]$ & 28 & 21 & 33 & 89 & 53 & 12 & 72 & 83 & 26 & 13 \\
\hline
\end{tabular}

\subsection{Comparison of the costs}

For constant core parameters all alternative configurations increase the complexity and costs of a DEMO reactor over a device based on the SND. Main capital cost drivers are the superconductors used for the TF and the PF coils. Comparing the length of the required superconductor strand can, therefore, be used as a lowest order indication of the cost increases.

As the cross-sectional area of the TF coil winding pack is independent of the TF coil shape, the length of the required TF coil conductor strand is proportional to the poloidal circumference of each TF coil, $L_{\mathrm{TF}}$. While XD, SFD and DND can be designed with similar TF coil volume, $V_{\mathrm{TF}}$, and $L_{\mathrm{TF}}$ as the SND configuration, Table 3 , the SXD increases $L_{\mathrm{TF}}$ and, hence, the costs of the TF coils by approximately $15 \%$, Fig. 3.

The length of the PF coil conductors increases with the radius of each coil and the maximum current in each coil. As the PF coil currents change during the discharge the maxima are not necessarily simultaneously obtained. Using the maximum current at the start or the end of the current flattop (SOF and EOF) indicates that the SXD and SFD configurations require $50 \%$ and $40 \%$ more PF coil conductors, respectively, Fig. 3. 


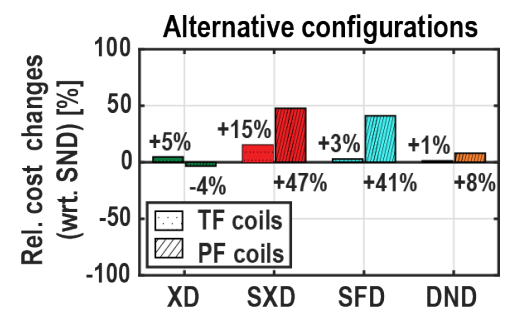

Figure 3. Comparison of the lowest order cost estimates for TF and PF coils in alternative configurations with the SND configuration. The estimates are based on the required conductor lengths stated in Table 3.

\subsection{Adaptation to a new baseline design}

To evaluate the implications of changes in the baseline design the reference configuration described in Section 3.2 as well as all assessed alternatives described in Section 3.3 were revised to consider changes in the reference parameters of the EU DEMO baseline design [49]. The changes comprise most notably a reduction of the number of TF coils from 18 to 16 and a reduction of the magnetic field from $5.8 \mathrm{~T}$ to $4.9 \mathrm{~T}$, while meeting all of the above described constraints. To keep the same net electric power output, the plasma elongation $\kappa_{95}$ is increased from 1.55 to 1.65 and $\beta_{\mathrm{N}}$ from 2.6 to 2.9. The changes in the specifications result in a plasma with a somewhat larger major radius of $R_{0}=8.9 \mathrm{~m}$ and a somewhat lower plasma current of $I_{\mathrm{P}}=19.1 \mathrm{MA}$. The resulting "2018" configurations are shown in Fig. 4.
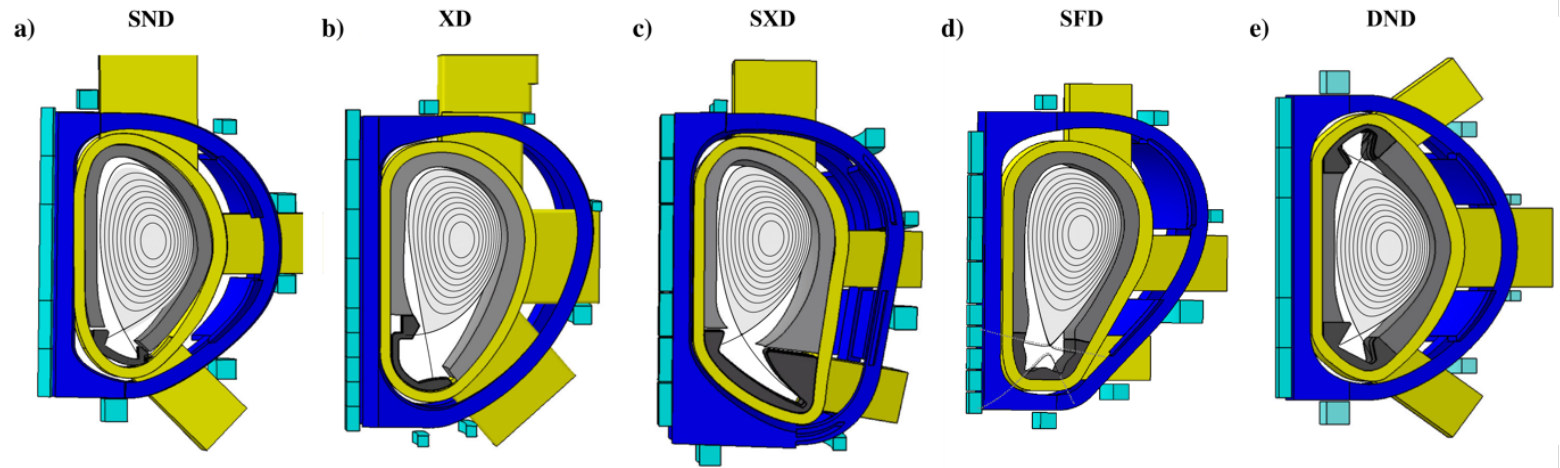

Figure 4. "2018” DEMO configurations featuring (a) a SND, (b) XD, (c) SXD, (d) SFD and (e) DND. The descriptions include 3D models of the blanket (light grey), the divertor modules (dark grey), a vacuum vessel including ports (yellow), toroidal field coils (blue) and poloidal field coils (cyan).

The revised plasma parameters lead to an approximately $15 \%$ larger plasma volume in all configurations. Due to the reduction of the number of TF coils the volume they encompass grows 10-15\% more than the plasma volume. This increase does, however, not affect the conclusion on the increased cost of the alternative configurations relative to the baseline. The revision of the configurations has also only led to minor changes of geometric parameters that are deemed to affect the exhaust performance (e.g. the connection length, flux flaring or target radii).

\subsection{Remote handling aspects}

Remote handling of in-vessel components such as the required periodic replacement of blanket modules and divertor cassettes will affect the availability of a reactor and, hence, the price of electricity and should, therefore, be included in the costs of alternative divertor configurations. To identify potential implications of the alternative configurations on the remote handling 3D models of the devices based on the " 2018 " configuration, introduced in Section 4.4, have been developed [49]. In addition to detailed models of the divertor cassettes that meet the interface and space requirements determined in the DEMO baseline activity [50], the " 2018 " configurations also include the first wall, a vacuum vessel with ports, discrete TF coils, their inter-coil structure and PF coils. Critical issues identified in the 3D analysis fed back into the 2D description of the configurations.

The SND configuration, Fig. 4(a), features a divertor port, which is inclined by $45^{\circ}$ with respect to the horizontal plane. Access to the blanket modules is provided via a top port and a vertical maintenance scheme [51].

The XD configuration, Fig. 4(b), offers similar access to the divertor as the SND, but requires larger divertor cassettes, which complicates remote handling. As for the SND access to the blanket modules is provided via a top port and a vertical maintenance scheme. 
The SXD configuration, Fig. 4(c), may feature a divertor port that has only a $10^{\circ}$ inclination with respect to the horizontal plane and whose dimensions are limited by the outer intercoil structure required to cope with higher electromagnetic loads expected in this configuration. The divertor cassette is even larger than the XD cassette further complicating remote access. Access to the blanket is provided via a top port and a vertical maintenance scheme.

The divertor coils of the SFD configuration, Fig. 4(d), restrict access to the divertor. A smaller port, which also competes with intercoil structure, offers horizontal access that is further complicated by the large SFD cassettes with four targets. Access to the blanket is provided via a top port.

The DND configuration, Fig. 4(e), features up-down symmetric divertor ports for divertor access that is similar to the baseline configuration. Access to the blanket must be provided by equatorial ports, which complicates both blanket segmentation and, consequently, remote handling operations.

\subsection{Outstanding issues and next steps}

Clearly, further constraints that will also have an impact upon the achievable geometric variations of the divertor configurations exist. A range of outstanding issues is identified and will be addressed in next steps.

(1) The structural integrity of the proposed TF coils, which presently deviate from typical D shaped coils, must be investigated and the coil shape adapted as necessary.

(2) The controllability of the vertical position and of the divertor configuration including the grazing angle of the field lines at the targets and the strike point location must be verified.

(3) The impact of the possible port locations and divertor module geometry on the costs of remote maintenance must be quantified.

(4) The potential benefits of additional PF coils based on copper conductors that could be placed inside the TF coils should be assessed.

\section{PREDICTION OF THE DIVERTOR PERFORMANCE}

A range of boundary models with varying degrees of complexity have been used to predict the effects of the alternative configurations on exhaust performance. Desired effects include an easier access to detachment, which is deemed necessary to obtain acceptable conditions at the plasma-wall interface in the divertor. This will have to be achieved largely through an increase of divertor radiation while avoiding excessive core confinement degradation. Once detachment is achieved the cold front should be reluctant to move along the divertor leg as it may lead to excessive core confinement degradation and a decrease of the neutral pressure in the pump ducts. The operating parameter range between detaching the divertor and the cold front reaching the X-point is commonly referred to as the detachment window and characterises the ability to handle transients. The alternative configurations are assessed through a comparison to the baseline solution. Such a comparison reduces the effect of systematic errors, which inevitably affect absolute predictions.

In order to evaluate the power exhaust performance of alternative divertors several figures of merit based on the constraints discussed in Section 2 are proposed. Avoiding excessive target erosion and operating in a detached regime, both, require that the electron temperature at the target is sufficiently low, with $T_{\mathrm{e}, \mathrm{t}}^{\max }=5 \mathrm{eV}$ being here used as a typical number [14]. In addition, the peak heat flux must not exceed the heat removal capacity of the target, with $q_{\perp, \mathrm{t}}^{\max }=5 \mathrm{MW} / \mathrm{m}^{2}$ being a typical value for the axisymmetric heat flux in charged particles.

(1) Required radiation fraction: The key concern in any extrapolation to DEMO is the required increase in the radiation fraction outside the LCFS, i.e. in the divertor, $f_{\text {rad,div }}$, and alternative configurations can be evaluated by their ability to decrease the required radiation fraction, $f_{\text {rad,div }}^{\text {req }}=f_{\text {rad,div }} \mid T_{\mathrm{e}, \mathrm{t}} \leq 5 \mathrm{eV} \wedge$ $q_{\perp, \mathrm{t}} \leq 5 \mathrm{MW} / \mathrm{m}^{2}$. Such a decrease of $f_{\mathrm{rad} \text { div }}^{\mathrm{req}}$ can be achieved by increasing the tolerable power that can be exhausted at the divertor target, $P_{\text {tar }}^{\text {tol }}=\left(1-f_{\text {rad,div }}^{\text {req }}\right) P_{\text {sep }}$. This first metric, therefore, quantifies the ability to exhaust more power at the target.

(2) Required impurity concentration: In addition to decreasing $f_{\text {rad,div }}^{\text {req }}$ alternative configurations can also reduce the impurity concentration required to achieve a desired radiation fraction and, hence, tolerable target temperatures. Since the key concern is excessive core radiation and fuel dilution, it is ultimately the ability to reduce the seed impurity (e.g. Ar) concentration required to cool the divertor, $c_{\mathrm{z}}^{\text {req }}=$ $c_{\mathrm{z}} \mid T_{\mathrm{e}, \mathrm{t}} \leq 5 \mathrm{eV} \wedge q_{\perp, \mathrm{t}} \leq 5 \mathrm{MW} / \mathrm{m}^{2}$ that determines the power exhaust performance.

It is expected that lowering $f_{\mathrm{rad}, \mathrm{div}}^{\mathrm{req}}$ and, ultimately, $c_{\mathrm{z}}^{\text {req }}$ would correspondingly increase the operating range to lower separatrix density, which would, for example be advantageous in increasing current drive efficiencies. 


\subsection{Required radiation fraction}

\subsubsection{Extended 2-point model}

The extended 2-point model can be used to relate upstream to target parameters [32,52]. To assess the ability to access detachment pressure losses along field lines are neglected. Assuming furthermore that effective volume losses due to cross-field transport are small, the parallel heat flux at the target is [52],

$$
q_{\|, \mathrm{t}}=\left(1-f_{\text {rad,div }}\right) \frac{B_{\text {tot, } \mathrm{t}}}{B_{\mathrm{tot}, \mathrm{u}}} q_{\|, \mathrm{u}} \approx\left(1-f_{\text {rad,div }}\right) \frac{R_{\mathrm{u}}}{R_{\mathrm{t}}} q_{\|, \mathrm{u}}
$$

For the investigated configurations the error introduced by approximating the ratios of the total magnetic fields with the inverse of the major radii is lower than $1 \%$. Note that at constant grazing angle at the target, $\alpha_{\mathrm{t}}$, the $R_{\mathrm{t}}$ dependence of the fluxtube cross-sectional area becomes exact. The parallel heat flux at the target and, hence $q_{\perp, \mathrm{t}}$, can be reduced by increasing the major radius of the target, $R_{\mathrm{t}}$. The tolerable power at the target $P_{\mathrm{tar}}^{\text {tol }} \propto 1-$ $f_{\text {rad,div }}^{\text {req }}$ that reduces the heat flux below $q_{\|, \mathrm{t}}^{\max }$, therefore, increases with $R_{\mathrm{t}}$. While the omission of cross-field transport in Eq. (3) is not justified at the low plasma temperatures in front of the target and usually referred to as divertor spreading (assumed to be of the order of a factor 3 in Section 2), the resulting dependence of $P_{\operatorname{tar}}^{\text {tol }} \propto 1-$ $f_{\text {rad,div }}^{\text {req }}$ on $R_{\mathrm{t}}$ is consistent with a divertor spreading that is independent of the divertor geometry.

Among the assessed configuration only the SXD promises a significant advantage with $R_{\mathrm{t}}$ of the outer divertor and the corresponding $P_{\mathrm{tar}}^{\text {tol }}$ increasing by $\sim 30 \%$ with respect to the SND, Table 4 . However, since the DND configuration deviates power from an inner to an outer target the corresponding $R_{\mathrm{t}}$ and, hence, $P_{\mathrm{tar}}^{\text {tol }}$ increase by $28 \%$ with respect to the inner target of the SND.

An increase in connection length only helps to cool the divertor to tolerable electron temperatures. Assuming that parallel heat transport is dominated by electron heat conduction, the target temperature is [52],

$$
T_{\mathrm{e}, \mathrm{t}} \propto\left(1-f_{\mathrm{rad}, \mathrm{div}}\right)^{2} /\left(L_{\|}^{4 / 7} R_{\mathrm{t}}^{2}\right),
$$

and the tolerable target power $P_{\text {tar }}^{\text {tol }} \propto 1-f_{\text {rad,div }}^{\text {req }}$ that cools the divertor below $T_{\mathrm{e}, \mathrm{t}}^{\mathrm{max}}$, hence, scales as $\propto L_{\|}^{2 / 7} R_{\mathrm{t}}$. The tolerable target power of the SXD should, thereby increase by more than $50 \%$ over the SND with the advantages of the SFDs only being slightly lower, Table 4 . The advantage of the DND is severely decreased as $L_{\|}$to an outer target of the DND is much shorter than to an inner target of the SND.

TABLE 4. Extended 2-point model predictions of the target power for tolerable conditions at the outer target, $P_{\mathrm{tar}}^{\mathrm{tol}}$, compared to the SND. The fraction of $P_{\text {sep }}$ exhausted in the outer divertor is assumed to be independent of the divertor configuration. Increases and decreases by more than $20 \%$ are highlighted in green and red (none), respectively.

\begin{tabular}{ccccccc}
\hline Criterion & $\boldsymbol{P}_{\mathrm{tar}}^{\mathrm{tol}} / \boldsymbol{P}_{\mathrm{tar}}^{\mathrm{tol}, \text { SND }}$ & XD & SXD & SFD(+) & SFD(- & DND \\
\hline$q_{\|, \mathrm{t}} \leq q_{\|, \mathrm{t}}^{\max }$ & $R_{\mathrm{t}} / R_{\mathrm{t}}^{\text {SND }}$ & 0.91 & 1.30 & 1.07 & 0.81 & 0.98 \\
\hline$T_{\mathrm{e}, \mathrm{t}} \leq T_{\mathrm{e}, \mathrm{t}}^{\max }$ & $\left(L_{\|}^{2 / 7} R_{\mathrm{t}}\right) /\left(L_{\|}^{S N D^{2 / 7}} R_{\mathrm{t}}^{S N D}\right)$ & 1.09 & 1.53 & 1.43 & 1.45 & 0.93 \\
\hline
\end{tabular}

Changes in the divertor configuration may affect the in-out power sharing. Recent power sharing measurements in attached TCV plasmas with various divertor configurations [53] are qualitatively consistent with a power sharing arising from the simultaneous application of the 2-point model to inner and outer divertor [54], which predicts,

$$
\frac{P_{\text {in }}}{P_{\text {out }}}=\frac{\mathcal{L}_{\|, \text {out }}}{\mathcal{L}_{\|, \text {in }}},
$$

where $\mathcal{L}_{\|} \equiv \int_{\text {upstream }}^{\text {target }} R_{0} / R(s) d s$ is a weighted connection length. While the stagnation point of the heat transport in the SOL would be a better correspondence to the upstream location of the two point model, it should, due to the strong ballooning character of cross field transport, only vary weakly among different divertor configuration. This assessment, therefore, continues to use the outboard midplane as the 'upstream' location. Considering the changes in the power sharing due to the changes in the divertor configuration in the predictions for $P_{\mathrm{tar}}^{\mathrm{tol}}$ is advantageous for all outer targets, albeit at the expense of the inner targets, Table 5. The redistribution of the challenge from the outer to the inner divertor is particularly large for XD and SFD(-) configuration and the smallest for the $\mathrm{SFD}(+)$. As the inner divertor in the SND usually represents the smaller challenge some redistribution may be acceptable or even desirable, e.g. to detach the inner and outer divertors at the same time.

${ }^{* *}$ The $\mathrm{SFD}(-)$ has a X-point separation of $d R_{\mathrm{u}, \mathrm{X} 2}=1 \mathrm{~mm}$. 
TABLE 5. Extended 2-point model predictions with variable power sharing of the target power for tolerable conditions at the inner and outer targets, $P_{\text {tar }}^{\text {tol }}$, compared to the SND. Increases and decreases by more than $20 \%$ are highlighted in green and red, respectively.

\begin{tabular}{cccccccccccc}
$\boldsymbol{P}_{\text {tar }}^{\text {tol }} / \boldsymbol{P}_{\text {tar }}^{\text {tol,SND }}$ & \multicolumn{2}{c}{ XD } & \multicolumn{2}{c}{ SXD } & \multicolumn{2}{c}{ SFD(+) } & \multicolumn{2}{c}{ SFD(-) $)^{\S}$} & \multicolumn{2}{c}{ DND } \\
\cline { 2 - 12 } Criterion & in & out & in & out & in & out & in & out & top & bot \\
\hline$q_{\|, \mathrm{t}} \leq q_{\|, \mathrm{t}}^{\max }$ & 0.64 & 1.14 & 0.76 & 1.54 & 0.84 & 1.16 & 0.58 & 1.26 & 0.88 & 1.29 \\
\hline$T_{\mathrm{e}, \mathrm{t}} \leq T_{\mathrm{e}, \mathrm{t}}^{\max }$ & 0.66 & 1.37 & 0.79 & 1.80 & 1.05 & 1.55 & 0.68 & 2.25 & 0.71 & 1.22 \\
\hline
\end{tabular}

\subsubsection{D fluid models}

A systematic study of the alternative configurations and the SND reference is carried out using the divertor transport codes TECXY and SOLEDGE2D-Eirene. Both codes are fluid codes that use ad-hoc cross-field diffusivities. The diffusion coefficients $D_{\perp, \mathrm{e} / \mathrm{i}}=0.42 \mathrm{~m}^{2} / \mathrm{s}$ and $\chi_{\perp, \mathrm{e} / \mathrm{i}}=0.18 \mathrm{~m}^{2} / \mathrm{s}$ were chosen to result in an upstream SOL width of approximately $3 \mathrm{~mm}$ for the single-null configuration and in attached conditions. This is significantly larger than the expected value (see Section 2) resulting in optimistic absolute numbers. The interpretation of the simulation will thus continue to focus on the relative performance with respect to the reference scenario.

Since simulations of DEMO-size configurations with medium-Z impurity, such as argon (Ar) are computationally expensive, the power crossing the separatrix is reduced with respect to the nominal value as a proxy for an increasing impurity radiation fraction, $P_{\text {sep }}^{\text {sim }}=\left(1-f_{\text {imp,div }}\right) P_{\text {sep. }}$. All scans are performed with a fixed separatrix density (at the stagnation point), $n_{\mathrm{e}, \mathrm{sep}}=2.5 \times 10^{19} \mathrm{~m}^{-3}$, corresponding to $\sim 30 \%$ of $\left\langle n_{\mathrm{e}}\right\rangle$ of the reference scenario, Section 2 .

In most cases the requirement on $T_{\mathrm{e}, \mathrm{t}}$ is found to be more severe than the requirement on $q_{\perp, \mathrm{t}}$. Since $q_{\perp, \mathrm{t}}$ depends strongly on $\alpha_{\mathrm{t}}$, Eq. (1), this may reverse, if a conservative value of $\alpha_{\mathrm{t}}$ constrains the configurations (see Section 2). The ability to meet either requirement is, therefore, discussed separately.

\subsubsection{TECXY}

The TECXY code [55] can treat diverted geometries with a single X-point and was recently extended to include the private flux region in the computational domain. The code simplifies the target geometry by assuming a perpendicular incidence of the flux surfaces. It also uses an analytic model for neutral particles. The perpendicular heat flux at the target is deduced from the grazing angle of the field line and the calculated parallel heat flux, $q_{\perp, \mathrm{t}}=q_{\|, \mathrm{t}}^{\mathrm{TECXY}} \sin \alpha_{\mathrm{t}}$. The model enhances the 2-point model by coupling flux tubes and assuming realistic spatial gradients and connection lengths. The TECXY simulations, thereby, add the effect of the divertor geometry on the competition between parallel and perpendicular transport. Its applicability is limited once the interaction with neutrals becomes significant. It is applied to the SND, XD and SXD configurations described in Section 3.1 and 3.2. In addition, it is applied to a $\mathrm{SFD}(+)$ with a somewhat larger separation of the X-points than the SFD configuration described in Section 3.2, limiting the extent of the considered PFR to the region between primary and secondary X-point.

The TECXY code has been used to vary the power crossing the separatrix. For the nominal value of $P_{\text {sep }}=150 \mathrm{MW}$, and without impurity seeding, the peak target temperatures and heat fluxes are not tolerable for all configurations. Reducing $P_{\text {sep }}$ reduces $q_{\perp, \mathrm{t}}$, Fig. 5(a,b), and $T_{\mathrm{e}, \mathrm{t}}$, Fig. 6(a,b), at both targets. Linear interpolation and in some cases extrapolation yields estimates of $P_{\text {sep }}^{\text {tol }}$ for both requirements.

As expected from the 2-point model, Sect. 4.1, all alternative configurations increase $P_{\text {sep }}^{\text {tol }}$ at the outer target for both criteria with the increase being largest for the SXD, Figs. 5(d) and 6(d). The expected decreases of $P_{\text {sep }}^{\text {tol }}$ at the inner target, however, only persists for the XD configuration. As expected the SFD leads to the largest $P_{\text {sep }}^{\text {tol }}$ at the inner target, Figs. 5(c) and 6(c). The beneficial effects of all alternatives are greater for the requirement on $T_{\mathrm{e}, \mathrm{t}}$ than for the requirement on $q_{\perp, \mathrm{t}}$.

According to the TECXY simulations the SXD configuration yields a larger beneficial effect for the outer target than $\mathrm{XD}$ and $\mathrm{SFD}(+)$, while the $\mathrm{SFD}(+)$ promises beneficial effects for both targets. 


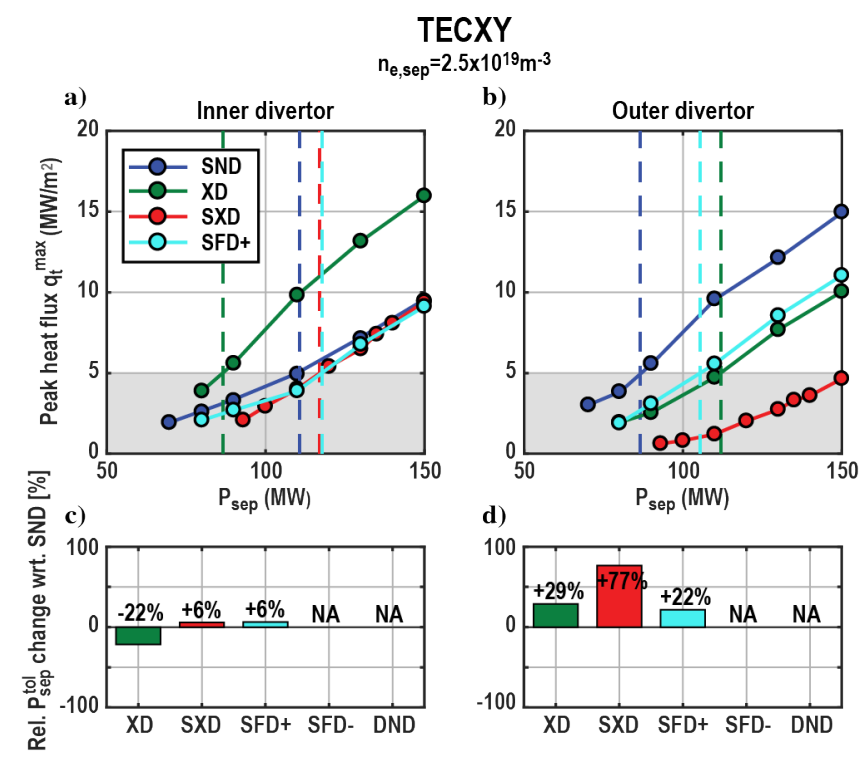

Figure 5. (a,b) Peak heat flux onto the targets $q_{\perp, t}$ resulting from TECXY calculations of pure deuterium and varying the power that crosses the separatrix in various configurations. The shaded regions indicate acceptable $q_{\perp, t}$ and the dashed line the interpolated tolerable $P_{\text {sep }}(c, d)$ Relative change of $P_{\text {sep }}^{\text {tol }}$ with respect to the SND configuration.

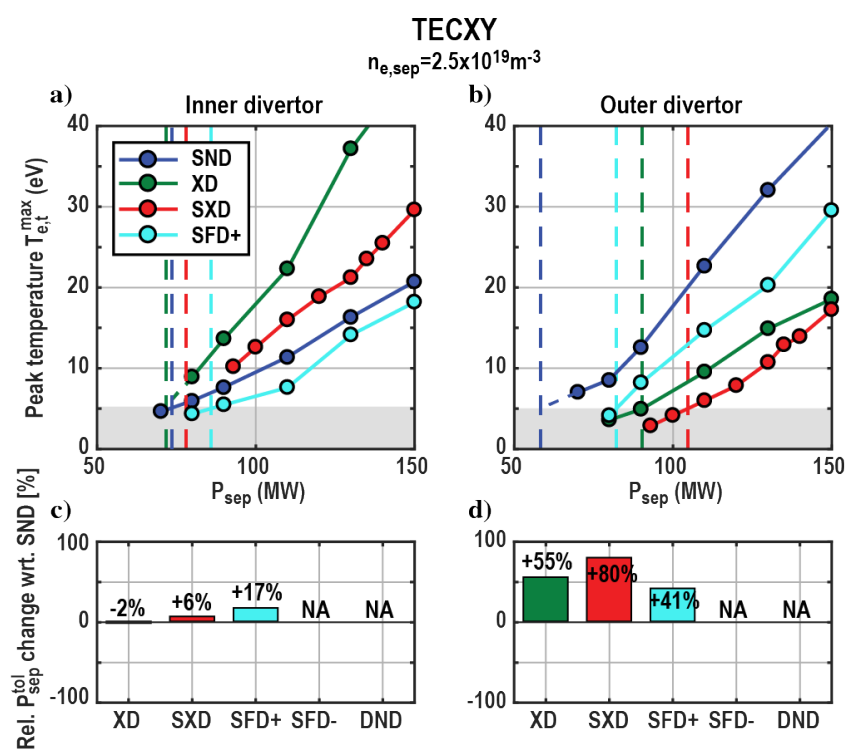

Figure 6. (a,b) Peak electron temperatures at the targets $T_{e, t}$ resulting from TECXY calculations of pure deuterium and varying the power that crosses the separatrix in various configurations. The shaded regions indicate acceptable $T_{e, t}$ and the dashed line the interpolated (and in some cases extrapolated) tolerable $P_{\text {sep }} \cdot(c, d)$ Relative change of $P_{\text {sep }}^{\text {tol }}$ with respect to the SND configuration.

\subsubsection{SOLEDGE2D}

Experiments and modelling have shown that the poloidal tilt of the target has a large effect on the detachment dynamics including the onset of detachment. This is caused by the reflection of recycling neutral into the SOL and the trapping of neutrals near the target. The realism of the simulation is, therefore, improved with the SOLEDGE2D code [56], which includes the target tilt and a kinetic treatment of neutrals through coupling with EIRENE [57]. In addition to the improved treatment of neutrals SOLEDGE2D can also simulate divertors with multiple X-points including SFD(+), SFD(-) and DND configurations.

The SOLEDGE2D-Eirene code has been used to vary the power crossing the separatrix [58]. As in the TECXY simulations reducing $P_{\text {sep }}$ generally reduces $q_{\perp, \mathrm{t}}$, Fig. 7(a,b), and $T_{\mathrm{e}, \mathrm{t}}$, Fig. 8(a,b), at both targets. 


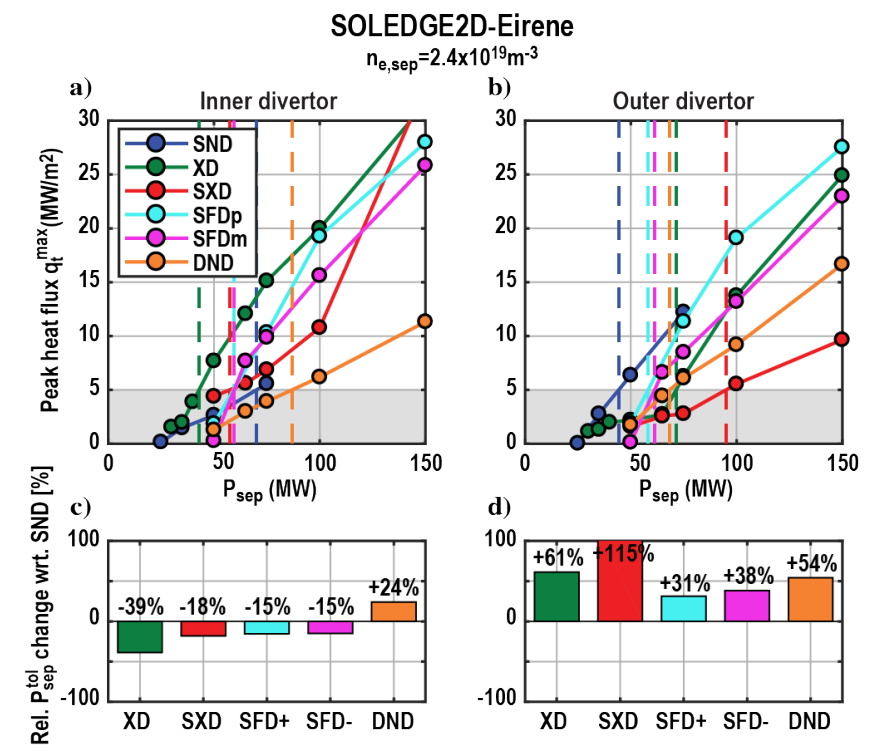

Figure 7. (a,b) Peak heat flux onto the targets $q_{\perp, t}$ resulting from SOLEDGE2D calculations of pure deuterium and varying the power that crosses the separatrix in various configurations. The shaded regions indicate acceptable $q_{\perp, t}$ and the dashed line the interpolated tolerable $P_{\text {sep. }}(c, d)$ Relative change of $P_{\text {sep }}^{\text {tol }}$ with respect to the SND configuration.

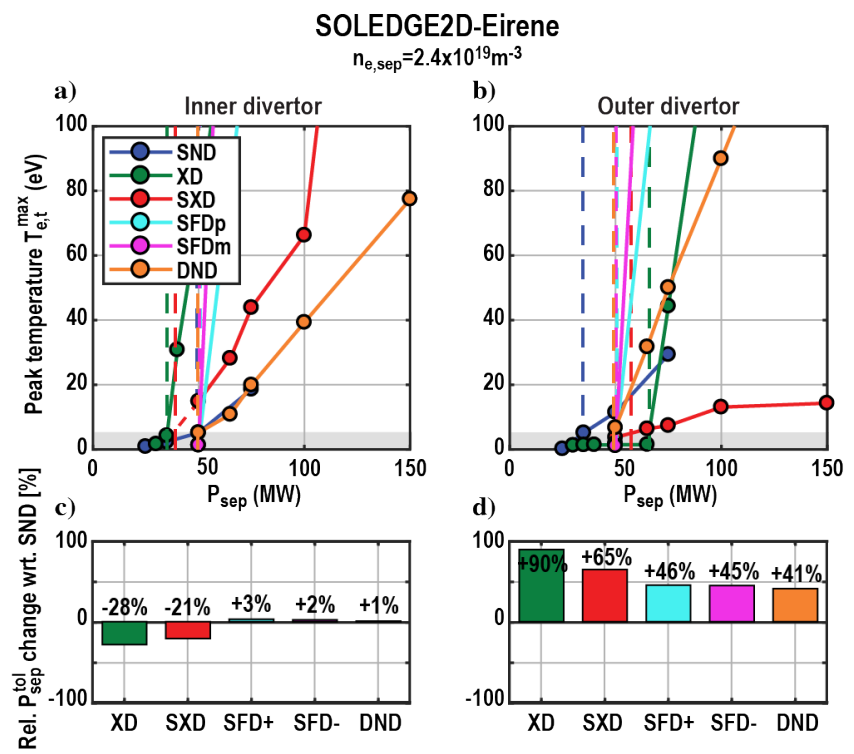

Figure 8. $(a, b)$ Peak electron temperatures at the targets $T_{e, t}$ resulting from SOLEDGE2D calculations of pure deuterium and varying the power that crosses the separatrix in various configurations. The shaded regions indicate acceptable $T_{e, t}$ and the dashed line the interpolated (and in some cases extrapolated) tolerable $P_{\text {sep. }}(c, d)$ Relative change of $P_{\text {sep }}^{\text {tol }}$ with respect to the SND configuration.

All alternative configurations increase $P_{\text {sep }}^{\text {tol }}$ at the outer target, Figs. 7(d) and 8(d). Absolute values, however, differ significantly from the TECXY predictions, Section 4.2.1. In particular, the in-out asymmetry in XD, SXD and $\operatorname{SFD}(+)$ is stronger. The $\mathrm{SFD}(-)$ shows similar performance to the $\mathrm{SFD}(+)$. The $\mathrm{DND}$ is predicted to have advantageous performance of the outer target and no repercussions for the inner target that becomes a second outer target.

\subsubsection{Comparison of the models}

The changes in the required divertor radiation fraction must be deduced from the relative changes of the estimates for $P_{\text {tar }}^{\text {tol }} \propto 1-f_{\text {rad,div }}^{\text {req }}$, which are obtained either directly or through its proxy $P_{\text {sep }}^{\text {tol }}$ from the physics models discussed in Sections 4.1.1 and 4.1.2. The predicted changes with respect to the SND baseline are summarised in Fig. 9. The considered physics models increase in complexity with the SOLEDGE2D-Eirene representing the most complete set of effects. There is a general trend towards increasing $1-f_{\text {rad,div }}^{\text {req }}$ by up to a factor of two, 
albeit in the cases of XD and SXD at the expense of the conditions at the inner target, presumably due to changes in the in-out power sharing. The beneficial effect of all alternative configurations except for the DND on the temperature is larger than the effect on the peak heat flux, presumably due to longer connection lengths. However, each additional effect can, to a somewhat lesser degree, still modify the advantage or disadvantage.
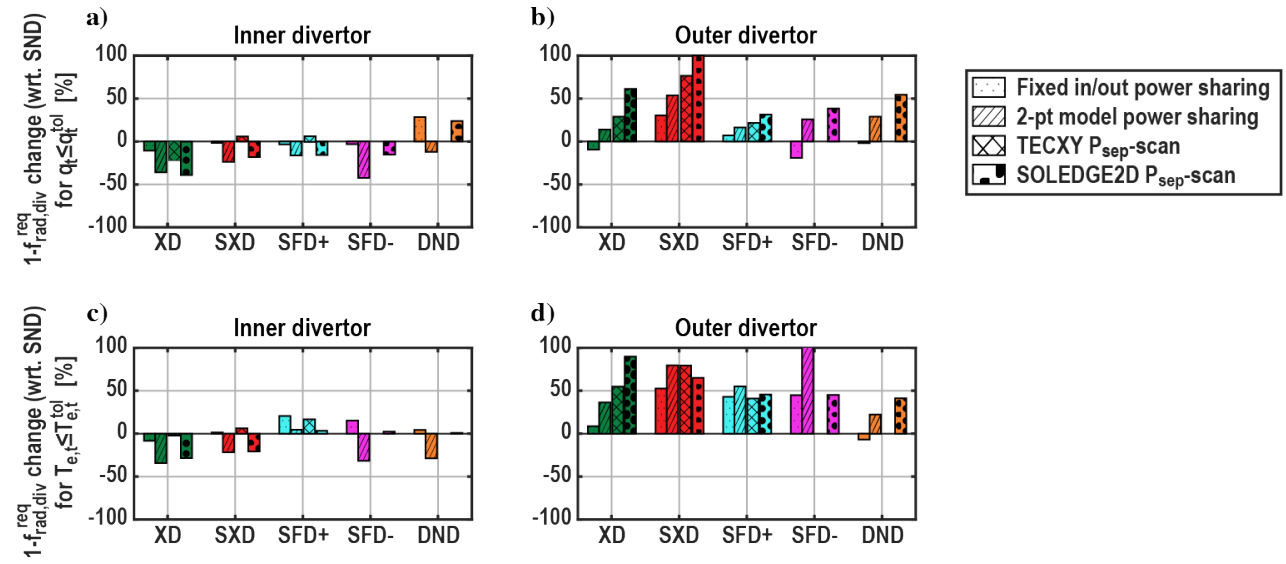

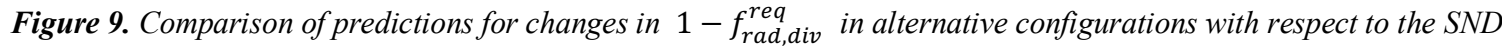
baseline for acceptable target heat flux $(a, b)$ and target electron temperature $(c, d)$ using physics models of increasing complexity.

As the required divertor radiation fraction $f_{\text {rad,div }}^{\text {req }}$ is high, a large relative increase of $1-f_{\text {rad,div }}^{\text {req }}$ is needed for any significant reduction of the required divertor radiation $P_{\text {rad,div }}^{\text {req }}$ as,

$$
\frac{\Delta P_{\text {rad }}^{\text {req }}}{P_{\text {rad }}^{\text {req }}}=-\frac{1-f_{\text {rad,div }}^{\text {req }}}{f_{\text {rad,div }}^{\text {req }}} \frac{\Delta\left(1-f_{\text {rad,div }}^{\text {req }}\right)}{1-f_{\text {rad,div }}^{\text {req }}} .
$$

With $f_{\text {req }}^{\text {req }}$ of the baseline scenario expected to be as high as $96 \%$ (as discussed in Section 2) an increase of $1-f_{\text {rad,div }}^{\text {req }}$ by a factor of 2 , decreases $P_{\text {rad,div }}^{\text {req }}$ according to Eq. (6) by only $\sim 4 \%$, Fig. 10 . The improved exhaust performance expected from the physics effects discussed so far is, therefore, likely not sufficient to justify the increased costs. Alternative configurations must, therefore, be largely based on their ability to increase the divertor radiation without detrimental effects on core performance.

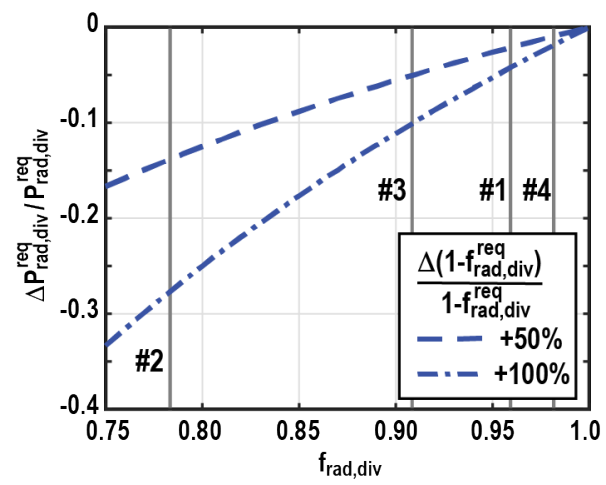

Figure 10. Dependence of the relative change in required divertor radiation $P_{\text {rad,div }}^{\text {req }}$ for two relative increases of 1 $f_{\text {rad,div }}^{\text {req }}$ on the divertor radiation fraction. The radiation fractions of the four scenarios of Table 2 are indicated.

\subsection{Required impurity concentration}

The TECXY code has also been used to simulate Ar seeding. The dynamics of all its charge states, which are treated as separate fluids with the same temperature as the main ions, is determined by the same plasma equations as the main ion species. The gas is injected as neutral Ar from a poloidal outer wall segment in the proximity of the X-point. However, due to its high recycling coefficient the solution is insensitive to the seeding location with neutral Ar and low ionization generally accumulating close to the divertor targets. The calculations are carried out for the nominal separatrix density, $n_{\mathrm{e}, \mathrm{sep}}=2.5 \times 10^{19} \mathrm{~m}^{-3}$, and power crossing the LCFS, $P_{\mathrm{sep}}=150 \mathrm{MW}$. Increasing the seeding rate increases the impurity concentration as well as the divertor radiation and reduces $q_{\perp, \mathrm{t}}$, Fig. 11(a,b), and $T_{\mathrm{e}, \mathrm{t}}$, Fig. 11(c,d), at both targets. The simulations are stopped when the electron temperature at the target decrease below $3 \mathrm{eV}$, where the physics model of the code is no longer applicable. In the case of the 
SXD, which has the lowest target temperatures without seeding, Section 5.1.2.1, this happens already for negligible seeding rates.

The effective charge of the plasma at the separatrix, $Z_{\text {eff,sep }}$, is used as a measure for the detrimental effect on the core performance. Both simulated alternatives, $\mathrm{XD}$ and $\mathrm{SFD}(+)$, require a lower $Z_{\text {eff,sep }}$ to obtain tolerable conditions at the outer target, Fig. 11(c,d). The calculations for the SND fail well before tolerable conditions are reached and no credible estimated of $Z_{\mathrm{eff}, \mathrm{rep}}^{\text {ree }}$ can be obtained. The better performance of the outer divertor of XD and SFD compared to the SND is nevertheless qualitative consistent and, hence, expected from the discussion of $P_{\text {res }}^{\text {tol }}$, Section 5.1. At the inner target the SFD $(+)$ performs similarly to the SND, whereas the simulations of the $\mathrm{XD}$ result in a higher $Z_{\mathrm{eff}, \mathrm{rep}}^{\mathrm{req}}$, Fig. 11(a,b), again consistent with the discussion of $P_{\text {res }}^{\text {tol }}$, Section 5.1, and understood as a consequence of the redistribution of exhaust power from the outer to the inner divertor.
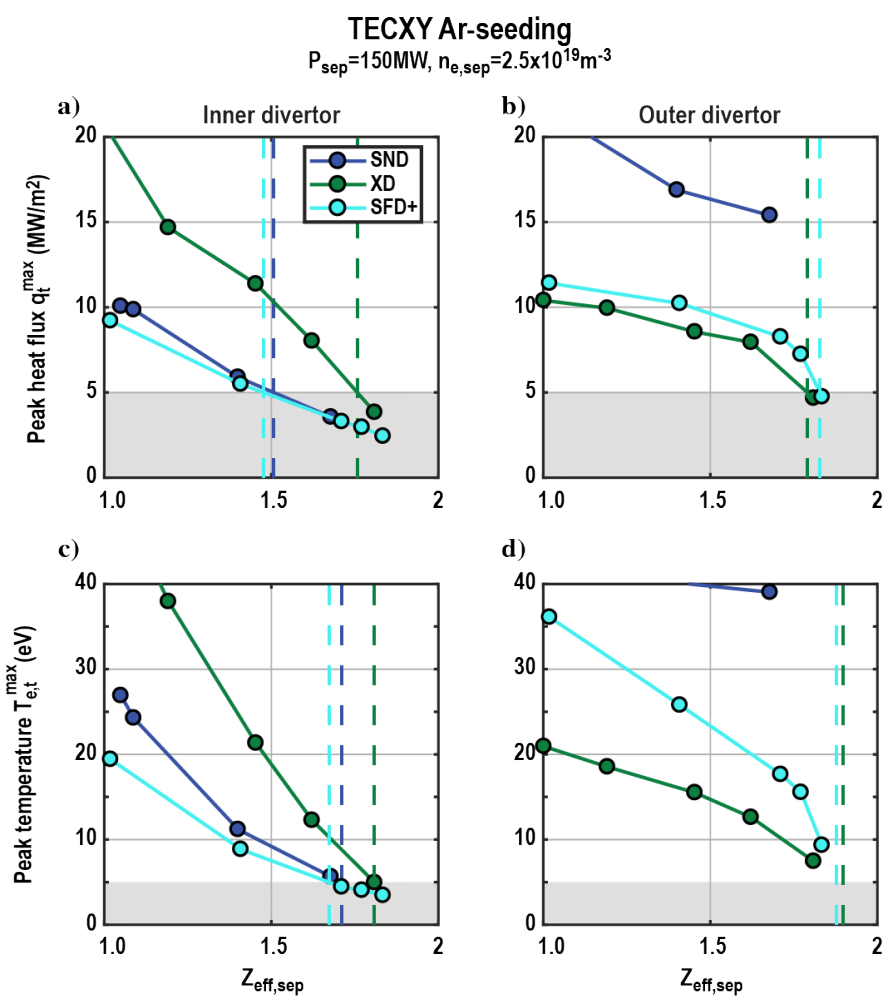

Figure 11. (a,b) Peak heat flux onto the targets $q_{\perp, t}$ and $(c, d)$ peak electron temperatures at the targets $T_{e, t}$ resulting from TECXY calculations of Ar seeding in various configurations. The shaded regions indicate acceptable $q_{\perp, t}$ and $T_{e, t}$ and the dashed line the interpolated tolerable $Z_{\text {eff,sep }}$.

In addition to decreasing the required radiation fraction better divertor performance would also be obtained by increasing the divertor radiation for the same impurity concentration. The simulations yield that XD and $\operatorname{SFD}(+)$,

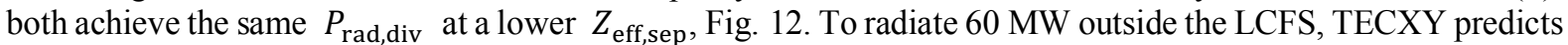
that the $\mathrm{XD}$ and $\mathrm{SFD}(+)$ require a $\sim 20 \%$ lower $Z_{\text {eff,sep }}$ than the SND. A more consequential comparison will, however, require the extension of the SND calculations towards acceptable conditions at both targets.

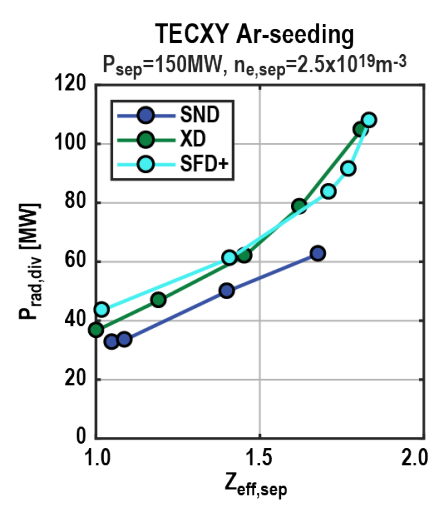

Figure 12. Predicted dependence of the radiated power on the effective charge number at the separatrix. 


\subsection{Outstanding issues and next steps}

The divertor simulations have, to date, only been able to partially evaluate the proposed figures of merit for the power exhaust performance. In particular a complete analysis of $f_{\text {rad,div }}^{\text {req }}$ and increasing $P_{\text {rad,div }}$ to address the figure of merit on the required impurity concentration is still outstanding. The simulations have also not yet addressed the width of the detachment window, which characterises the ability of an exhaust solution to handle transients and which is expected to be a key advantage of the XD and SXD configurations. The assessment must, finally, also address the coupling of the radiative divertor to the core.

One of the main outstanding elements in the assessment remains the inclusion of predictive models for the crossfield transport. While the inclusion of drifts is, in principle, possible and faces mainly numerical challenges, the physics basis of the turbulent cross-field transport remains to be established.

Next steps of the assessment of alternative divertor configurations for DEMO must also include their particle exhaust performance as the divertor geometry has a large effect on the neutral compression in the divertor and, hence the location and size of the vacuum pumps. The inclusion of a pumping solution will likely require a further iteration of the $2 \mathrm{D}$ and $3 \mathrm{D}$ configurations.

\section{CONCLUSION}

Obtaining tolerable conditions at the divertor targets in DEMO will require a higher fraction of power that is dissipated in the divertor volume as well as a greater pressure drop along magnetic field lines in the SOL than necessary in today's devices. Since it is not certain that the required high radiative power exhaust is compatible with the simultaneously required core performance, alternative magnetic configurations to the conventional single-null divertor are assessed. The assessment is limited to alternative divertor concepts that rely on the same technologies and are compatible with the same core scenario envisaged for the current baseline DEMO.

DEMO configurations with XD, SXD, SFD and DND plasma exhaust solutions that rely exclusively on PF coils outside the TF coils have been developed. The PF coils meet constraints on forces, current densities and magnetic fields that are compatible with existing technologies. The main encountered limitations are that XD and SXD features can only be applied to the outer divertor. In addition, the flux flaring at the outer target of the XD is limited by the minimum grazing angle of field lines at the target and the major radius of the outer target of the SXD by coil forces. SFD and DND can be fully implemented, but may be affected by control limitations. A possible reduction of the flux swing can be largely eliminated by a greater segmentation of the CS. Capital cost drivers are larger TF coils in the case of the SXD, with approximately $15 \%$ higher TF coil costs than the SND baseline, and larger PF coils in the case of SXD and SFD, with approximately $50 \%$ higher PF coil costs. In addition, alternative configurations will complicate the remote maintenance of the divertor cassettes and blanket modules, but a quantification of the complexity increases in terms of cost increases remains to be established.

Performance improvements are expected with regard to a lower divertor radiation fraction required to obtain acceptable conditions at the target, $f_{\text {rad,div }}^{\text {req }}$, and a higher radiation fraction achieved with the same impurity concentration. All alternative configurations reduce the radiation fraction required for acceptable conditions at the outer target, but XD and SXD achieve this partially on the expense of less favourable conditions at the inner target. However, the overall high values of $f_{\text {rad,div }}^{\text {req }}$ entail that the expected increases of the tolerable residual power of up to a factor of two only translate into modest reductions of $f_{\text {rad,div }}^{\text {req }}$ of the order of $5 \%$. The main advantage of alternative configurations must therefore be their ability to increase the divertor radiation without degrading core performance. First fluid simulations of Ar seeding in a subset of the assessed configurations indicate advantages of the XD and SFD configurations to radiate more power at the same impurity concentration. A quantitative comparison requires further optimization of the target geometry including baffling as well as the inclusion of an improved and self-consistent model for turbulent transport.

The assessment has, to date, only addressed the lowest order engineering constraints and most main physics aspects. It has not encountered any show stoppers, but identifies key limitations of XD and SXD. Within these limitations it nevertheless confirms advantageous exhaust performance of all assessed alternative configurations. A quantitative cost-benefit calculation, however, requires a more detailed engineering analysis and further physics model development and validation.

\section{ACKNOWLEDGEMENTS}

This work has been carried out within the framework of the EUROfusion Consortium and has received funding from the Euratom research and training programme 2014-2018 and 2019-2020 under grant agreement No 633053. The views and opinions expressed herein do not necessarily reflect those of the European Commission. This work was supported in part by the Swiss National Science Foundation. HR would like to specifically thank B.P. Duval 
and C. Theiler along with the entire boundary group at the SPC for stimulating discussions that greatly helped to shape this assessment.

\section{REFERENCES}

[1] ROMANELLI, F., "Fusion Electricity - A roadmap to the realisation of fusion energy", EFDA, 2012, http://www.eurofusion.org/wpcms/wp-content/uploads/2013/01/JG12.356-web.pdf

[2] DONNE, T, MORRIS, W., "European Research Roadmap to the Realisation of Fusion Energy", EUROfusion, 2018, https://www.euro-fusion.org/eurofusion/roadmap/

[3] WENNINGER, R., et al., Nucl. Fusion 54 (2014) 114003.

[4] REINKE, M.L, Nucl. Fusion 57 (2017) 034004.

[5] GOLDSTON, R., REINKE, M.L, SCHWARTZ, J.A., Plasma Phys. Control. Fusion 59 (2017) 055015.

[6] SOUKHANOVSKII, V.A., Plasma Phys. Control. Fusion 59 (2017) 064005.

[7] WENNINGER, R., et al., Nucl. Fusion 55 (2015) 063003.

[8] MARTIN, Y, TAKIZUKA, T. and ITPA CDBM H-mode Threshold Database Working Group, J. Phys. 123 (2008) 012033.

[9] PITTS, R., et al, Nucl. Mater. Energy 20 (2019) 100696, DOI: 10.1016/j.nme.2019.100696.

[10] EICH, T., et al., Phys. Rev. Lett. 107 (2011) 215001.

[11] GOLDSTON, R.J., Nucl. Fusion 52 (2012) 013009.

[12] YOU, J.H., et al., Nucl. Mater. Energy 16 (2018) 1.

[13] PITTS, R.A., et al., Nucl. Mater. Energy 12 (2017) 60.

[14] STANGEBY, P.C., LEONARD, A.W., Nucl. Fusion 51 (2011) 063001.

[15] LIPSCHULTZ, B., et al., Fusion Sci Technol. 51 (2007) 369.

[16] CHANG, C.S, et al., Nucl. Fusion 57 (2017) 116023.

[17] FEDERICI, G., et al., Fus. Eng. Design 89 (2014) 882-889

[18] FEDERICI, G., et al., Nucl. Fusion 59 (2019) 066013.

[19] KOTSCHENREUTHER, M., et al., Phys. Plasmas 14 (2007) 072502.

[20] KOTSCHENREUTHER, M., et al., Phys. Plasmas 20 (2013) 102507.

[21] COVELE, B., et al., Nucl. Fusion 57 (2017) 086017.

[22] SHIMOMURA, Y., KEILHACKER, M., LACKNER, K., MURMANN, H., Nucl. Fusion 23 (1983) 869.

[23] PITTS, R.A., et al., J. Nucl Mater. 290 (2001) 940.

[24] THEILER, C., et al., Nucl. Fusion 57 (2017) 072008.

[25] VALANJU, P.M., et al., Phys. Plasmas 16 (2009) 056110.

[26] HUTCHINSON, I., Nucl. Fusion 34 (1994) 1337.

[27] LIPSCHULTZ, B., et al., Nucl. Fusion 56 (2016) 056007.

[28] FISHPOOL, G., et al., J. Nucl. Mater. 438 (2013) S356.

[29] LABOMBARD, B., et al., Nucl. Fusion 55 (2015) 053020.

[30] UMANSKY, M.V., et al., Phys. Plasmas 24 (2017) 056112.

[31] MOULTON, D., HARRISON, J., LIPSCHULTZ, B., COSTER, D., Plasma Phys. Control. Fusion 59 (2017) 065011.

[32] PETRIE, T.W., et al., Nucl. Fusion 53 (2013) 113024.

[33] RYUTOV, D.D., Phys. Plasmas 14 (2007) 064502.

[34] RYUTOV, D.D., SOUKHANOVSKII, V.A., Phys. Plasmas 22 (2015) 110901.

[35] RYUTOV, D.D., et al., Phys. Scr. 89 (2014) 088002.

[36] UMANSKY, M.V., RYUTOV, D.D., Phys. Plasmas 23 (2016) 030701.

[37] REIMOLD, F., et al., Nucl. Fusion 55 (2015) 033004.

[38] RYUTOV, D.D., COHEN, R.H., ROGNLIEN, T.D., UMANSKY, M.V., Plasma Phys. Control. Fusion 54 (2012) 124050.

[39] LUNT, T., et al., Plasma Phys. Control. Fusion 58 (2016) 045027.

[40] LABIT, B., et al., Nucl. Mater. Energy 12 (2017) 1015.

[41] PIRAS, F., et al., Plasma Phys. Control. Fusion 51 (2009) 055009.

[42] SOUKHANOVSKII, V.A., et al., J. Nucl. Mater. 415 (2011) S365.

[43] ALLEN S.L., et al., "Results from initial snowflake divertor physics studies on DIII-D", Proc. 24th IAEA FEC, San Diego, 8-13 October 2012, PD/1-2, http://www-naweb.iaea.org/napc/physics/FEC/FEC2012/html/proceedings.pdf

[44] REIMERDES, H., et al., Plasma Phys. Control. Fusion 55 (2013) 124027.

[45] SMICK, N., LABOMBARD, B., PITCHER, C.S. J. Nucl. Mater. 337 (2005) 281.

[46] BORTNIKOV, A.V., et al., “T-12 divertor experiment”, Plasma Physics and Controlled Nuclear Fusion Research 1980, Vol. I, Eighth Conference Proceedings, Brussels, 1-10 July 1980, X-2-2.

[47] MEYER, H., et al., Plasma Phys. Control. Fusion 47 (2005) 843. 
[48] AmbrosinO, R., CASTALdO, A., HA, S., LOSCHIAVO ,V.P., MERRIMAN, S. and REIMERDES, H., Fus. Eng. Des. 146 (2019) 2717.

[49] MARZULLO, D., et al., "Preliminary Engineering Assessment of Alternative Magnetic Divertor Configurations for EUDEMO”, $14^{\text {th }}$ International Symposium on Fusion Nuclear Technology, 22-27 September 2019, Budapest, Hungary, P1027.

[50] MARZULLO, D., et al., Fus. Eng. Des. 146 (2019) 942.

[51] LOVING, A., et al., Fus. Eng. Des. 89 (2014) 2246.

[52] STANGEBY, P.C., Plasma Phys. Control. Fusion 60 (2018) 044022.

[53] MAURIZIO, R., et al., Nucl. Fusion 58 (2018) 016052.

[54] MAURIZIO, R., et al., Nucl. Mater. Energy 19 (2019) 372.

[55] ZARGÓRSKI, R., GERHAUSER, H., Phys. Scr. 70 (2004) 173.

[56] BUFFERAND, H., et al., J. Nucl. Mater. 438 (2013) S445.

[57] REITER, D., et al., Fusion Sci. Technol. 47 (2005) 172.

[58] INNOCENTE, P., et al., "Modeling of power exhaust in DEMO alternative divertor configurations with SOLEDGE2DEIRENE", 23 ${ }^{\text {rd }}$ International Conference on Plasma Surface Interactions in Controlled Fusion Devices, Princeton, 17-22 June, 2018. 